\title{
Photoinduced Interfacial Electron Injection Dynamics in Dye- Sensitized Solar Cells under Photovoltaic Operating Conditions
}

\author{
Joël Teuscher, ${ }^{\dagger,}$ Jean-David Décoppet, ${ }^{\dagger}$ Angela Punzi, ${ }^{\dagger, \perp}$ Shaik M. Zakeeruddin, ${ }^{\dagger}$ Jacques-E. Moser, ${ }^{\dagger}$ \\ and Michael Grätzel* $*$
}

${ }^{\dagger}$ Photochemical Dynamics Group and ${ }^{\ddagger}$ Laboratory of Photonics and Interfaces, Institute of Chemical Sciences and Engineering, École polytechnique fédérale de Lausanne (EPFL), Station 6, CH-1015 Lausanne, Switzerland

\begin{abstract}
We report a pump-probe spectroscopy study of electron injection rates in dye-sensitized solar cell (DSSC) devices. We examine the case of working devices employing an N719 ruthenium sensitizer and an iodide electrolyte. Electron injection is found to occur mainly on a sub-100 fs time scale, followed by a slower component with a lifetime of $26.9 \mathrm{ps}$, in accordance with previous reports on model samples. The amplitude of this latter component varies with electrolyte composition from 25 to $9 \%$. The appearance of slower components in the electron injection dynamics may be attributed to an aggregated or weakly bound state of the surface-adsorbed N719 sensitizer. Further measurements are reported varying the cell light bias and load conditions, revealing no influence on electron injection dynamics. No other electron injection event is found to occur up to $1 \mathrm{~ns}$. These results show no evidence for a slowdown of electron injection under working conditions compared to model systems for the electrolytes examined in this study.
\end{abstract}

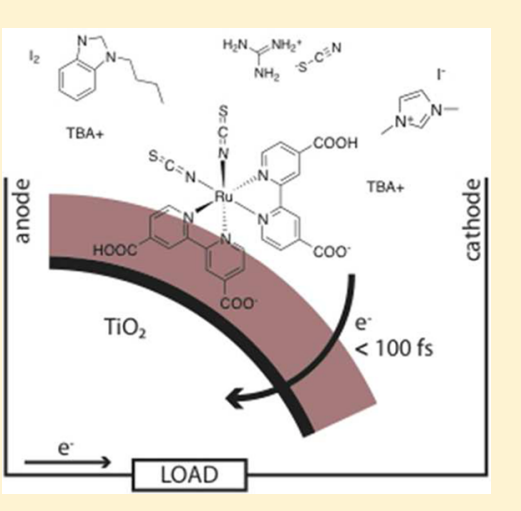

SECTION: Kinetics and Dynamics

$\mathrm{D}$ ye-sensitized solar cells (DSSCs) can efficiently convert solar radiation into electricity by means of a molecular or quantum dot light harvester anchored on a wide-band-gap mesoporous semiconductor film. Electron injection from the photoexcited light harvester into the conduction band of the semiconductor, typically $\mathrm{TiO}_{2}$, is the first reaction in the photoconversion process. ${ }^{1}$ Ruthenium(II) polypyridyl complexes, such as $\mathrm{N} 719\left(\left(\mathrm{Bu}_{4} \mathrm{~N}\right)_{2}\left[\mathrm{Ru}(\mathrm{dcbpyH})_{2}(\mathrm{NCS})_{2}\right]\right)$, adsorbed on mesoporous nanocrystalline titania films have been successfully used as sensitizers in these devices. ${ }^{2}$ They have, therefore, been widely used as model systems for the investigation of electron injection dynamics.

Pump-probe spectroscopy allows monitoring of the rise of the oxidized dye formed after electron injection. Multiexponential kinetic behavior is observed, rendering difficult the application of a simple kinetic model to readily describe these dynamics. N719 and its close derivatives are known to inject electrons into $\mathrm{TiO}_{2}$ with close to unit quantum yield. ${ }^{3}$ Dynamics of charge injection from ruthenium complexes in $\mathrm{TiO}_{2}$ have been widely studied for the past decades. ${ }^{4-14}$ The kinetics observed were reported as being multiphasic and composed of an ultrafast component in the 10-100 fs time range and of a slower exponential part occurring in the 10-100 ps time range. Dynamics are dependent on the sample environment and preparation. Benkö et al. interpreted the fast and the slow components in terms of electron injection from the singlet and triplet states, respectively. ${ }^{6,7}$ Wenger et al. extended this model by ascribing the observed multiphasic dynamics to inhomogeneity in dye adsorption on the substrate. $^{15}$ The slower time component was assigned to loosely bound dye molecules, present in the aggregated form, which undergo weaker electronic coupling with the $\mathrm{Ti}(3 \mathrm{~d})$ states of the $\mathrm{TiO}_{2}$ conduction band and therefore inject from their triplet metal-to-ligand charge-transfer state $\left({ }^{3} \mathrm{MLCT}\right)$. By contrast, the strongly anchored dye molecules inject directly from their singlet excited state on a femtosecond time scale. This was supported by the observation of monoexponential electron-transfer kinetics with a rise time shorter than 20 fs for a sample where the dye has been adsorbed from a highly dilute solution containing fewer aggregates. This explanation was supported by ultrafast fluorescence studies that revealed a sub10 fs electron injection from the ${ }^{1}$ MLCT. ${ }^{16}$

The majority of studies involving the electron injection reaction have so far been performed on model systems. Often, injection dynamics were examined using dye-covered $\mathrm{TiO}_{2}$ films in vacuum, exposed to a gas or solvent, rather than on complete state-of-the-art devices. However, Haque et al. reported the influence of the electrolyte composition on the electron injection dynamics. ${ }^{17}$ Using N719 as a sensitizer, in conjunction with an electrolyte containing $0.1 \mathrm{M}$ lithium iodide, the devices showed a 20 times slower injection rate upon addition of $0.5 \mathrm{M}$ 4-tert-butylpyridine, indicating that electron injection from the singlet state was unlikely to contribute to photocurrent generation in high-performance devices that often employ these two additives. More recently, Koops et al. reported electron injection half-times up to $3 \mathrm{~ns}$,

Received: October 19, 2012

Accepted: November 30, 2012

Published: November 30, 2012 
dependent on the dye environment, by monitoring the transient emission dynamics, with an instrument response of 250 ps. ${ }^{18,19}$ Furube et al. investigated DSSCs at open circuit by means of ultrafast diffuse reflectance transient absorption. ${ }^{20} \mathrm{~A}$ direct correlation between the transient absorbance absolute amplitude and the incident photon to current conversion efficiency of the device was observed.

The goal of this investigation is to determine electron injection times in working devices by directly monitoring electron injection using pump-probe spectroscopy with a 100 fs instrument response. The effect of the composition of the electrolyte on the interfacial electron injection dynamics was investigated, along with the influence of the light bias and that of changing the external load resistance. We show that an electrolyte judiciously designed to yield optimal photovoltaic performance exhibits also the smallest amplitude for slower components of electron injection, probably by reducing dye aggregation.

The photovoltaic performance of reference devices was assessed by measuring the photocurrent-voltage $(\mathrm{J} / \mathrm{V})$ curves to confirm the properties of the samples, which were later used for transient absorbance measurements. The samples studied were sealed devices of varying electrolyte composition, and their fabrication is described in the Experimental Section. Cells 1 and 2 are standard working devices of the same composition, using electrolyte Z946 (1.0 M 1,3-dimethylimidazolium iodide (DMII), $0.15 \mathrm{M} \mathrm{I}_{2}, 0.5 \mathrm{M} \mathrm{N}$-butylbenzimidazole (NBB), and $0.1 \mathrm{M}$ guanidinium thiocyanate (GNCS) in 3-methoxypropionitrile (MPN)). Cell 3 contains no NBB in its electrolyte, and its $P V$ parameters were not measured. The fourth cell studied (cell 4) contains a mixture of $0.5 \mathrm{M} \mathrm{NBB}$ in MPN, and cell 5 contains pure MPN as the solvent. These last two devices produce no photocurrent because they lack the iodide/iodine redox couple. Control experiments using films of titania only with and without electrolyte contact have also been performed.

Photocurrent-voltage curves of cells 1 and 2 are shown in Figure 1. These cells exhibit efficiencies of 3.90 and $3.98 \%$

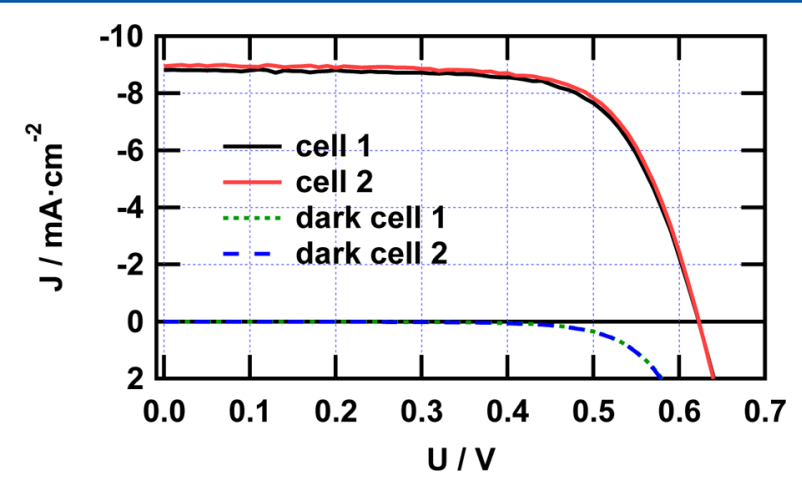

Figure 1. Photocurrent-voltage curves of cells 1 and 2, with $3.5 \mu \mathrm{m}$ thick $\mathrm{TiO}_{2}$ transparent mesoporous films, sensitized with $\mathrm{N} 719$ and volatile electrolyte Z946 under full $100 \mathrm{~mW} \mathrm{~cm}^{-2}$ simulated AM 1.5 sunlight and in the dark.

under 1 sun illumination with $V_{\text {oc }}$ of 622 and $623 \mathrm{mV}$, shortcircuit current densities of 8.8 and $8.9 \mathrm{~mA} \mathrm{~cm}^{-2}$, and fill factors of 69.9 and $70.4 \%$, respectively. The reported efficiencies are lower than those of state-of-the-art devices fabricated with N719 because we employed thin $(3.5 \mu \mathrm{m}) \mathrm{TiO}_{2}$ films without additional scattering layers so that the samples would remain transparent for the pump-probe experiment. The observed short-circuit photocurrent densities of $8.5-9 \mathrm{~mA} \mathrm{~cm}^{-2}$ are close to the value expected for such thin $\mathrm{TiO}_{2}$ films and N719 as a sensitizer.

We employed pump-probe transient absorbance spectroscopy to study the electron injection dynamics using working DSSCs with different electrolyte compositions. Electron transfer was probed at $860 \mathrm{~nm}$, a wavelength that allows convenient monitoring of the transient absorbance of the oxidized dye with a contribution of less than $15 \%$ originating from the excited sensitizer species. ${ }^{4,6,15}$ Monochromatic probe light was used for detection rather than white light in order to avoid chirp-induced artifacts and ensure the fastest time response along with a precise determination of time zero. Excitation of the dye by the pump laser pulse (see eq 1) was followed by electron injection in the $\mathrm{TiO}_{2}$ conduction band (eq 2 ). Therefore, monitoring the appearance of the transient absorbance of the oxidized dye adequately reflects the electron injection dynamics.

$$
\begin{aligned}
& \mathrm{SITiO}_{2}+h v \rightarrow \mathrm{S}^{*} \mid \mathrm{TiO}_{2} \\
& \mathrm{~S}^{*}\left|\mathrm{TiO}_{2} \rightarrow \mathrm{S}^{+}\right| \mathrm{TiO}_{2}+\mathrm{e}_{\mathrm{cb}}^{-}
\end{aligned}
$$

All samples showed an ultrafast rise within a time scale shorter than the instrument response function (IRF), accounting for the majority of the total signal amplitude. This rapid signal rise is followed by a slower component, whose amplitude varies from sample to sample, as shown in Figure 2a.

Figure $2 b$ shows normalized data, over a longer time scale of 1 ns. No significant change in the signal amplitude was observed after $100 \mathrm{ps}$, indicating that the electron injection is completed by that time. Normalization of the data for these five devices was simplified by using a singular value decomposition (SVD) procedure, allowing for a reduction in the noise level and, thus, granting easier fitting convergence. They were then fitted globally using eq 3, a sum of convolutions of an exponential with a Gaussian, representing the IRF, adapted from van Stokkum et al. ${ }^{21}$ Here, $\sigma$ represents the width of the Gaussian, and $\mu$ is a correction for the position of time zero, while $\tau_{i}$ are the time constants. We typically used $n=2$, with $\tau_{2}$ fixed at an infinite time corresponding to $1 \mu \mathrm{s}$ on our time scale. $\tau_{1}$ and its relative amplitude are therefore the parameters of interest.

$$
\begin{aligned}
\Delta A(t)= & \sum_{i=1}^{n}\left(\frac{A_{i}}{2} \cdot \exp \left(-\frac{t}{\tau_{i}}\right) \cdot \exp \left(\frac{1}{\tau_{i}} \cdot\left(\mu+\left(\frac{\sigma^{2}}{2 \cdot \tau_{i}}\right)\right)\right) .\right. \\
& \left.\left(1+\operatorname{erf}\left(\frac{t-\left(\mu-\frac{\sigma^{2}}{\tau_{i}}\right)}{\sqrt{2} \cdot \sigma}\right)\right)\right)
\end{aligned}
$$

Results of the fit of eq 3 are summarized in Table 1. $\sigma$ is shorter than the measured instrument response, implying that part of the rise lies within the laser pulse and confirming the femtosecond time scale of electron injection. The rise time $\tau_{1}$ is derived as 26.9 ps for the five samples, a value that matches reported literature values for slower components of electron injection. ${ }^{12}$ The amplitude of this component, $A_{1}$, varies from 24.5 down to $9.0 \%$ of the total amplitude, being maximal in pure MPN solvent. Upon addition of $0.5 \mathrm{M} \mathrm{NBB}$, the amplitude is decreased to $13.0 \%$. Apparently, the addition of NBB reduces dye aggregation or causes a reorganization of the 

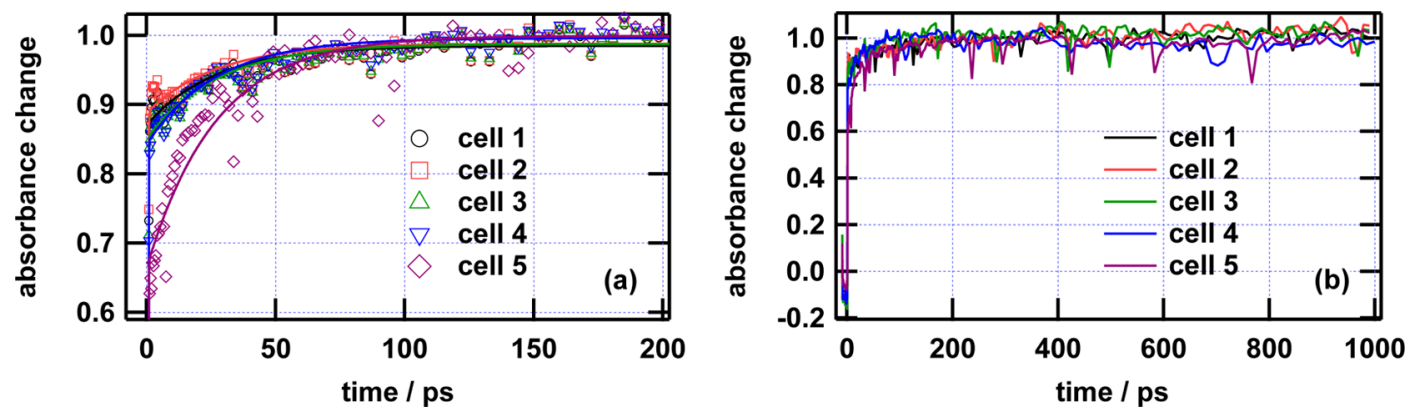

Figure 2. Normalized transient absorbance measurements at $860 \mathrm{~nm}$ following ultrafast laser pulse excitation at $530 \mathrm{~nm}$ of cells $1-5$ in dark conditions. Cell 1: Z946; cell 2: Z946; cell 3: Z946 without NBB; cell 4: 0.5 M NBB in MPN; and cell 5: MPN. Dye: N719. (a) Traces reflect the transient after SVD simplification, and solid lines correspond to a fit by a convolution function of a Gaussian instrument response and two exponential rises. (b) Lines are raw data recorded over 1 ns.

Table 1. Results of the Fit from Equation 3 with $n=2^{a}$

\begin{tabular}{|c|c|c|c|c|c|c|}
\hline cell & $\sigma(\mathrm{ps})$ & $\mu(\mathrm{ps})$ & $A_{1} \%$ & $\tau_{1}(\mathrm{ps})$ & $A_{2} \%$ & $\tau_{2}(\mathrm{ps})$ \\
\hline 1. Z946 & & & $10.1 \pm 0.5$ & & $89.9 \pm 0.5$ & \\
\hline 2. Z946 & & & $9.0 \pm 0.5$ & & $91.0 \pm 0.5$ & \\
\hline 3. Z946 without $\mathrm{NBB}$ & 0.038 & 0.88 & $11.9 \pm 0.5$ & $26.9 \pm 1.0$ & $88.1 \pm 0.5$ & $1 \times 10^{6}$ \\
\hline 4. NBB in MPN & & & $13.0 \pm 0.5$ & & $87.0 \pm 0.5$ & \\
\hline 5. MPN & & & $24.5 \pm 0.4$ & & $75.5 \pm 0.5$ & \\
\hline
\end{tabular}

dye at the nanoparticle surface, increasing the strongly bound fraction of N719 and explaining the dominance of the faster injection from the dye singlet state. The use of electrolyte without NBB exhibits an amplitude value close to that of cell 4, suggesting that the other components of the electrolyte such as DMII, iodine, and GSCN likewise have a beneficial effect on the dye adsorption and therefore coupling to the surface. Devices containing state-of-the-art Z946 electrolyte exhibit an even lower fraction of slow electron injection because its amplitude value decreases to $9.0 \%$. These results support the notion that the appearance of slower components in the electron injection dynamics is linked to an aggregated or weakly bound state of the surface-adsorbed N719 sensitizer, in accordance with the different injection time constants reported in the literature. ${ }^{4-11}$ This is in keeping with the large variation in the relative amplitudes of these two kinetic components reported by different groups.

However, our results appear to be at variance with the findings of Haque et al. concerning also complete devices. ${ }^{17}$ These authors probed the oxidized dye appearance at 800 instead of $860 \mathrm{~nm}$, a wavelength at which the excited dye makes a relatively larger contribution to the transient absorption signal. The subtraction procedure used in this study can therefore lead to minimization of the ultrafast component of the electron injection versus the slower components. Results from Koops et al. ${ }^{18}$ that report even slower injection rates on the nanosecond time scale are likely to be biased by the emission analysis employed. The use of time-correlated singlephoton counting monitors the ${ }^{3} \mathrm{MLCT}$ lifetime and, thus, might report loosely bound or even free dye molecules in solution or aggregates that inject on a slower time scale than singlet states. The importance of the mode of adsorption and the configuration of the sensitizer for the electron injection dynamics is confirmed also by the study of Asbury et al. reporting a diminution in the amplitude of the slower components after aging of the sample. ${ }^{10}$ This supports the notion that slow reorganization of loosely bound dye molecules to a self-assembled monolayer occurs on the titania surface when put in contact with the electrolyte.

Further studies examined the effect of illumination and applying an external load on the electron injection dynamics. The light source was a halogen lamp, calibrated to a fluence of $106 \mathrm{~mW} \mathrm{~cm}^{-2}$ at the sample position. Cell 1 was then either short-circuited or left at open circuit. A $20 \mathrm{ohm}$ load resistor was likewise added to the external circuit in order to study its impact on the reaction dynamics. According to the $I / V$ data reported above, this load resulted in a $40 \mathrm{mV}$ voltage drop across the load.

Figure 3 shows that the time response of the transient absorption due to the appearance of the oxidized sensitizer is

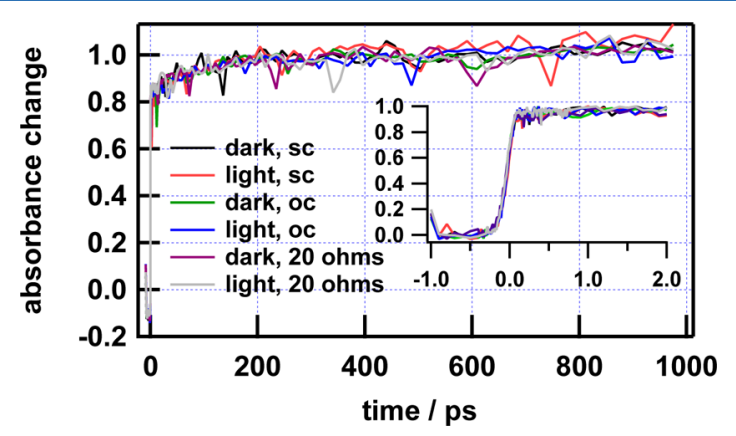

Figure 3. Normalized transient absorbance measured at $860 \mathrm{~nm}$ after ultrafast laser pulse excitation at $530 \mathrm{~nm}$ of cell 1 in the dark and under illumination with a $106 \mathrm{~mW} \mathrm{~cm}{ }^{-2}$ halogen lamp at different loads. The inset shows the signal at around time zero.

practically the same for all of the conditions investigated. At open circuit, a maximum voltage is built up across the cell, increasing the concentration of electrons and their Fermi energy to the highest level. The transient absorbance change corresponding to each devices' specific load superimposes perfectly, and it can be therefore concluded that the concentration of conduction band electrons within the range 
investigated here does not affect the injection dynamics. Measurements at the shortest times, at approximately time zero, are shown in the inset of Figure 3. The rise of the oxidized dye signal confirms that no difference is witnessed even on the fastest time scales, electron injection being of mainly in the sub$100 \mathrm{fs}$ time range. These measurements are in accordance with those of Koops et al., who applied an electrical bias to working devices while measuring their emission decays. This condition led to only a weak change in reported electron injection dynamics. $^{19}$

In conclusion, we have shown that the electron injection dynamics in working DSSCs are comparable to those measured on a single electrode in a pure solvent. The interfacial electron transfer from the excited ruthenium complex into the conduction band of $\mathrm{TiO}_{2}$ occurs mainly on a femtosecond time scale, with most of its amplitude lying under 100 fs. A slower component of electron injection is measured with a lifetime of $26.9 \mathrm{ps}$, in accordance with previous reports. This is attributed to electron injection from the ${ }^{3} \mathrm{MLCT}$ states of aggregates or loosely bound forms of the sensitizer molecules. No other electron injection component was found up to $1 \mathrm{~ns}$. The amplitude of the slower component depends on the dye's environment, an effect that has been probed by varying the electrolyte composition. A well-designed electrolyte decreases the amount of dye molecules that are injecting slowly, that is, from a thermalized triplet state. Moreover, there is no evidence for any slowdown of electron injection under working conditions such as illumination with a light bias at both short and open circuit or inserting a load in the external circuit.

\section{EXPERIMENTAL SECTION}

Photovoltaic devices are prepared using standard $\mathrm{TiO}_{2}$ films with a $3.5 \mu \mathrm{m}$ transparent mesoporous layer of $20 \mathrm{~nm}$ anatase particles on top of fluorine-doped tin oxide (FTO)-coated glass. Preparation of the paste has been described elsewhere. ${ }^{22}$ Films were dyed for $12 \mathrm{~h}$ in a solution of N719 dye $\left(5 \times 10^{-4}\right.$ $M)$. Cells were then sealed with a platinized FTO counter electrode using a polymer (Surlyn, Dupont) and subsequently filled with an electrolyte composed of 1.0 M 1,3-dimethylimidazolium iodide (DMII), $0.15 \mathrm{M} \mathrm{I}_{2}, 0.5 \mathrm{M} \mathrm{N}$-butylbenzimidazole (NBB), and 0.1 M guanidinium thiocyanate (GNCS) in 3methoxypropionitrile (MPN), coded Z946. The electrolyte composition was also modified to investigate the influence of its various constituents on electron injection dynamics. Devices were coated with a UV-cutoff/antireflecting polymer.

A $450 \mathrm{~W}$ xenon light source (Oriel, U.S.A.) was used to characterize the cells. The spectral output gave an irradiance of $100 \mathrm{~mW} \mathrm{~cm}^{-2}$ and was matched in the spectral region of 350$750 \mathrm{~nm}$ with the aid of a Schott K113 Tempax sunlight filter (Präzisions Glas \& Optik GmbH, Germany) in order to reduce the mismatch between the simulated and true solar spectra to less than $2 \%$. The current-voltage characteristics of the cell under these conditions were obtained by applying an external potential bias to the cell and measuring the generated photocurrent with a Keithley 2400 digital source meter (Keithley, U.S.A.). The devices were masked to attain an illuminated active area of $0.159 \mathrm{~cm}^{2}$.

The femtosecond transient spectrometer has been described elsewhere. ${ }^{23}$ We used it in this study in the two-color configuration. In short, an amplified Ti:Sapphire femtosecond laser at $1 \mathrm{kHz}$ (CPA-2001, Clark-MXR) sourced two NOPAs. The pump was tuned at $530 \mathrm{~nm}$ and the probe at $860 \mathrm{~nm}$. Typical instrument response measured by a Kerr gating technique in a thin glass window (SF10, $0.3 \mathrm{~mm}$ ) was $180 \mathrm{fs}$. Typical measurements were performed over $1 \mathrm{~ns}$, with 200 data points distributed on a logarithmic scale, with a smallest increment of $10 \mathrm{fs}$ close to time zero. Measurements were systematically repeated twice per sample, and the cells were excited from the electrolyte side of the device. The fluence on the cells was kept low, typically at $400 \mu \mathrm{J} \mathrm{cm}^{-2}$.

\section{AUTHOR INFORMATION}

\section{Corresponding Author}

*E-mail: michael.graetzel@epfl.ch.

\section{Present Address}

${ }^{\perp}$ Hach Lange $\mathrm{GmbH}$, Rte de Compois 6, CH-1222 Vésenaz, Switzerland.

\section{Notes}

The authors declare no competing financial interest.

\section{ACKNOWLEDGMENTS}

Financial support from the Swiss National Science Foundation is gratefully acknowledged. We thank Dr. Carole Grätzel and Dr. Robin Humphry-Baker (EPFL) for fruitful discussions.

\section{REFERENCES}

(1) O’Regan, B. C.; Grätzel, M. A Low-Cost, High-Efficiency SolarCell Based on Dye-Sensitized Colloidal $\mathrm{TiO}_{2}$ Films. Nature 1991, 353, 737-740.

(2) Nazeeruddin, M. K.; Zakeeruddin, S. M.; Humphry-Baker, R.; Jirousek, M.; Liska, P.; Vlachopoulos, N.; Shklover, V.; Fischer, C.; Grätzel, M. Acid-Base Equilibria of (2,2'-Bipyridyl-4,4'-dicarboxylic acid)ruthenium(II) Complexes and the Effect of Protonation on Charge-Transfer Sensitization of Nanocrystalline Titania. Inorg. Chem. 1999, 38, 6298-6305.

(3) Nazeeruddin, M. K.; Kay, A.; Rodicio, I.; Humphry-Baker, R.; Muller, E.; Liska, P.; Vlachopoulos, N.; Grätzel, M. Conversion of Light to Electricity by cis- $\mathrm{X}_{2} \mathrm{Bis}\left(2,2^{\prime}\right.$-bipyridyl-4,4'-dicarboxylate)ruthenium(II) Charge-Transfer Sensitizers $\left(\mathrm{X}=\mathrm{Cl}^{-}, \mathrm{Br}^{-}, \mathrm{I}^{-}, \mathrm{CN}^{-}\right.$, and $\left.\mathrm{SCN}^{-}\right)$on Nanocrystalline $\mathrm{TiO}_{2}$ Electrodes. J. Am. Chem. Soc. 1993, 115, 6382-6390.

(4) Tachibana, Y.; Moser, J.-E.; Grätzel, M.; Klug, D. R.; Durrant, J. R. Subpicosecond Interfacial Charge Separation in Dye-Sensitized Nanocrystalline Titanium Dioxide Films. J. Phys. Chem. 1996, 100, 20056-20062.

(5) Tachibana, Y.; Nazeeruddin, M. K.; Grätzel, M.; Klug, D. R.; Durrant, J. R. Electron Injection Kinetics for the Nanocrystalline $\mathrm{TiO}_{2}$ Films Sensitised with the Dye $\left(\mathrm{Bu}_{4} \mathrm{~N}\right)_{2} \mathrm{Ru}(\mathrm{dcbpyH})_{2}(\mathrm{NCS})_{2}$. Chem. Phys. 2002, 285, 127-132.

(6) Benko, G.; Kallioinen, J.; Korppi-Tommola, J. E. I.; Yartsev, A. P.; Sundström, V. Photoinduced Ultrafast Dye-to-Semiconductor Electron Injection from Nonthermalized and Thermalized Donor States. J. Am. Chem. Soc. 2002, 124, 489-493.

(7) Kallioinen, J.; Benko, G.; Sundström, V.; Korppi-Tommola, J. E. I.; Yartsev, A. P. Electron Transfer from the Singlet and Triplet Excited States of $\mathrm{Ru}(\mathrm{dcbpy})_{2}(\mathrm{NCS})_{2}$ into Nanocrystalline $\mathrm{TiO}_{2}$ Thin Films. J. Phys. Chem. B 2002, 106, 4396-4404.

(8) Kallioinen, J.; Benko, G.; Myllyperkio, P.; Khriachtchev, L.; Skarman, B.; Wallenberg, R.; Tuomikoski, M.; Korppi-Tommola, J. E. I.; Sundström, V.; Yartsev, A. P. Photoinduced Ultrafast Dynamics of $\mathrm{Ru}(\mathrm{dcbpy})_{2}(\mathrm{NCS})_{2}$-Sensitized Nanocrystalline $\mathrm{TiO}_{2}$ Films: The Influence of Sample Preparation and Experimental Conditions. J. Phys. Chem. B 2004, 108, 6365-6373.

(9) Myllyperkio, P.; Benko, G.; Korppi-Tommola, J. E. I.; Yartsev, A. P.; Sundström, V. A Study of Electron Transfer in Ru(dcbpy $)_{2}(\mathrm{NCS})_{2}$ Sensitized Nanocrystalline $\mathrm{TiO}_{2}$ and $\mathrm{SnO}_{2}$ Films Induced by RedWing Excitation. Phys. Chem. Chem. Phys. 2008, 10, 996-1002.

(10) Asbury, J.; Ellingson, R.; Ghosh, H. N.; Ferrere, S.; Nozik, A.; Lian, T. Femtosecond IR Study of Excited-State Relaxation and 
Electron-Injection Dynamics of $\mathrm{Ru}(\mathrm{dcbpy})_{2}(\mathrm{NCS})_{2}$ in Solution and on Nanocrystalline $\mathrm{TiO}_{2}$ and $\mathrm{Al}_{2} \mathrm{O}_{3}$ Thin Films. J. Phys. Chem. B 1999, 103, 3110-3119.

(11) Asbury, J.; Anderson, N.; Hao, E.; Ai, X.; Lian, T. Parameters Affecting Electron Injection Dynamics from Ruthenium Dyes to Titanium Dioxide Nanocrystalline Thin Film. J. Phys. Chem. B 2003, 107, 7376-7386.

(12) Ardo, S.; Meyer, G. J. Photodriven Heterogeneous Charge Transfer with Transition-Metal Compounds Anchored to $\mathrm{TiO}_{2}$ Semiconductor Surfaces. Chem. Soc. Rev. 2009, 38, 115-164.

(13) Listorti, A.; O'Regan, B. C.; Durrant, J. R. Electron Transfer Dynamics in Dye-Sensitized Solar Cells. Chem. Mater. 2011, 23, 3381-3399.

(14) Mahanta, S.; Furube, A.; Matsuzaki, H.; Murakami, T. N.; Matsumoto, H. Electron Injection Efficiency in Ru-Dye Sensitized $\mathrm{TiO}_{2}$ in the Presence of Room Temperature Ionic Liquid Solvents Probed by Femtosecond Transient Absorption Spectroscopy: Effect of Varying Anions. J. Phys. Chem. C 2012, 116, 20213-20219.

(15) Wenger, B.; Grätzel, M.; Moser, J.-E. Rationale for Kinetic Heterogeneity of Ultrafast Light-Induced Electron Transfer from $\mathrm{Ru}$ (II) Complex Sensitizers to Nanocrystalline $\mathrm{TiO}_{2}$. J. Am. Chem. Soc. 2005, 127, 12150-12151.

(16) Bräm, O.; Cannizzo, A.; Chergui, M. Ultrafast Fluorescence Studies of Dye Sensitized Solar Cells. Phys. Chem. Chem. Phys. 2012, 14, 7934-7937.

(17) Haque, S. A.; Palomares, E.; Cho, B.; Green, A.; Hirata, N.; Klug, D. R.; Durrant, J. R. Charge Separation versus Recombination in Dye-Sensitized Nanocrystalline Solar Cells: the Minimization of Kinetic Redundancy. J. Am. Chem. Soc. 2005, 127, 3456-3462.

(18) Koops, S. E.; Durrant, J. R. Transient Emission Studies of Electron Injection in Dye Sensitised Solar Cells. Inorg. Chim. Acta 2008, 361, 663-670.

(19) Koops, S. E.; O’Regan, B. C.; Barnes, P. R. F.; Durrant, J. R. Parameters Influencing the Efficiency of Electron Injection in DyeSensitized Solar Cells. J. Am. Chem. Soc. 2009, 131, 4808-4818.

(20) Furube, A.; Wang, Z.-S.; Sunahara, K.; Hara, K.; Katoh, R.; Tachiya, M. Femtosecond Diffuse Reflectance Transient Absorption for Dye-Sensitized Solar Cells under Operational Conditions: Effect of Electrolyte on Electron Injection. J. Am. Chem. Soc. 2010, 132, 66146615.

(21) van Stokkum, I. H. M.; Larsen, D. S.; van Grondelle, R. Global and Target Analysis of Time-Resolved Spectra. Biochim. Biophys. Acta 2004, 1657, 82-104.

(22) Wang, P.; Zakeeruddin, S. M.; Moser, J.-E.; Grätzel, M. A New Ionic Liquid Electrolyte Enhances the Conversion Efficiency of DyeSensitized Solar Cells. J. Phys. Chem. B 2003, 107, 13280-13285.

(23) Pelet, S.; Grätzel, M.; Moser, J.-E. Femtosecond Dynamics of Interfacial and Intermolecular Electron Transfer at Eosin-Sensitized Metal Oxide Nanoparticles. J. Phys. Chem. B 2003, 107, 3215-3224. 